\title{
POSITIVE VALUES OF INHOMOGENEOUS QUATERNARY OUADRATIC FORMS, II
}

\author{
VISHWA CHANDER DUMIR *
}

(Received 2 August 1966)

In a previous paper [4] we showed that $\Gamma_{3,1}=\frac{16}{3}$. For the definition of $\Gamma_{r, s}$ for an indefinite quadratic form in $n=r+s$ variables of the type $(r, s)$ see the above paper. Here we shall show that $\Gamma_{2,2}=16$. More precisely we prove:

THEOREM. Let $Q(x, y, z, t)$ be an indefinite quaternary quadratic form with determinant $D>0$ and signature $(2,2)$. Then given any real numbers $x_{0}, y_{0}, z_{0}, t_{0}$ we can find integers $x, y, z, t$ such that

$$
0<Q\left(x+x_{0}, y+y_{0}, z+z_{0}, t+t_{0}\right) \leqq(16|D|)^{\ddagger} .
$$

Equality is necessary if and only if either

$$
\begin{aligned}
& Q(x, y, z, t) \sim \rho Q_{1}=\rho(x y+z t) ; \quad \text { or } \\
& Q(x, y, z, t) \sim \rho Q_{2}=\rho\left(x^{2}-y^{2}-z^{2}+t^{2}\right) ; \quad \text { or } \\
& Q(x, y, z, t) \sim \rho Q_{3}=\rho\left(x^{2}-y^{2}-2 z t\right) ;
\end{aligned}
$$

where $\rho \neq 0$. For $Q_{1}$ equality occurs if and only if

$$
\begin{aligned}
& \left(x_{0}, y_{0}, z_{0}, t_{0}\right) \equiv(0,0,0,0)(\bmod 1), \text { for } Q_{2} \text { if and only if } \\
& \left(x_{0}, y_{0}, z_{0}, t_{0}\right) \equiv\left(\frac{1}{2}, \frac{1}{2}, \frac{1}{2}, \frac{1}{2}\right)(\bmod 1) \text { and for } Q_{3} \text { if and only if } \\
& \left(x_{0}, y_{0}, z_{0}, t_{0}\right) \equiv\left(\frac{1}{2}, \frac{1}{2}, 0,0\right)(\bmod 1) .
\end{aligned}
$$

I wish to express my deep sense of gratitude to Professor R. P. Bambah for his inspiring guidance and valuable suggestions at all stages of this work.

- This paper forms a part of the author's Ph.D. dissertation accepted at the Ohio State University, Columbus, Ohio. 


\section{Some lemmas}

In the course of the proof we shall use the following Lemmas:

Lемма 1. Let $Q(x, y, z, t)$ be an indefinite quaternary quadratic form of the type $(2,2)$ and determinant $D>0$. Then there exist integers $x_{1}, y_{1}$, $z_{1}, t_{1}$ such that

$$
0<Q\left(x_{1}, y_{1}, z_{1}, t_{1}\right) \leqq\left(\frac{81}{16} D\right)^{\frac{1}{4}}
$$

except when $Q(x, y, z, t) \sim \rho Q_{1}, \rho \neq 0$.

This is Theorem 1 of Oppenheim [6].

Leмma 2. Let $\varphi(y, z, t)$ be an indefinite ternary quadratic form with determinant $D<0$, then we can find integers $y_{2}, z_{2}, t_{2}$ such that

$$
0<\varphi\left(y_{2}, z_{2}, t_{2}\right) \leqq\left(\frac{9}{4}|D|\right)^{\frac{1}{3}}
$$

except when $\varphi(y, z, t) \sim \rho\left(y^{2}+z t\right), \rho>0$.

This is a theorem due to Oppenheim [5].

Leмma 3. Let $\varphi(y, z, t)$ be an indefinite ternary quadratic form with determinant $D<0$. Then given any real numbers $y_{0}, z_{0}, t_{0}$ we can find $(y, z, t) \equiv\left(y_{0}, z_{0}, t_{0}\right)(\bmod 1)$ such that

$$
0<\varphi(y, z, t) \leqq(4|D|)^{\frac{1}{3}} .
$$

This is the theorem of Barnes [1].

LemMa 4. Let $\varphi(y, z, t)$ be an indefinite ternary quadratic form with determinant $D \neq 0$, then given any real numbers $y_{0}, z_{0}, t_{0}$ we can find $(y, z, t) \equiv\left(y_{0}, z_{0}, t_{0}\right)(\bmod 1)$ satisfying

$$
|\varphi(y, z, t)| \leqq\left(\frac{27}{100}|D|\right)^{\frac{1}{5}} .
$$

This is due to Davenport [3].

Lemma 5. Let $\psi(z, t)$ be an indefinite binary quadratic form with discriminant $\Delta^{2}>0$ and $\lambda>0$ be a real number. Then given $z_{0}, t_{0}$ we can find $(z, t) \equiv\left(z_{0}, t_{0}\right)(\bmod 1)$ satisfying

$$
-\frac{\Delta}{4 \lambda} \leqq \psi(z, t)<\frac{\lambda \Delta}{4} .
$$

This is Theorem 1 of Blaney [2].

Lemma 6. Let $\psi(z, t)$ be an indefinite binary quadratic form with discriminant $\Delta^{2}>0$ and let $\infty \geqq \mu \geqq 3$ be a given real number. Then given $z_{0}, t_{0}$ we can find $(z, t) \equiv\left(z_{0}, t_{0}\right)(\bmod 1)$ satisfying 


$$
-\frac{\mu \Delta}{\{(1+\mu)(9+\mu)\}^{\frac{1}{2}}} \leqq \psi(z, t)<\frac{\Delta}{\{(1+\mu)(9+\mu)\}^{\frac{1}{2}}} .
$$

If $\mu=\infty$, equality occurs if and only if

$$
\begin{aligned}
& \psi(z, t) \sim c \psi_{1}(z, t)=c z t,\left(z_{0}, t_{0}\right) \equiv(0,0)(\bmod 1) ; \text { or } \\
& \psi(z, t) \sim c \psi_{2}(z, t)=c\left(z^{2}-t^{2}\right) ;\left(z_{0}, t_{0}\right) \equiv\left(\frac{1}{2}, \frac{1}{2}\right)(\bmod 1) ; c>0 .
\end{aligned}
$$

This is Theorem 2 of Blaney [2].

LeMma 7. Let $\psi(z, t)$ be an indefinite binary quadratic form with discriminant $\Delta^{2}>0$. Then given $\nu>1$ and any real numbers $z_{0}, t_{0}$ there exist $(z, t) \equiv\left(z_{0}, t_{0}\right)(\bmod 1)$ such that

$$
-\frac{v^{2} \Delta}{\left\{(\nu-1)^{3}(v+3)\right\}^{\frac{1}{2}}} \leqq \psi(z, t)<-\frac{\Delta}{\left\{(\nu-1)^{3}(\nu+3)\right\}^{\frac{1}{2}}} .
$$

This is Theorem 3 of Blaney [2].

\section{Proof of the Theorem}

Let

$$
m=\inf \{Q(x, y, z, t): x, y, z, t \text { integers, } Q(x, y, z, t)>0\}
$$

\subsection{CASE $m=0$}

Lemma 8. If $m=0$, then the theorem is true.

Proof. Since $m=0$; given $\varepsilon_{0}\left(0<\varepsilon_{0}<1\right)$ we can find integers $x_{1}, y_{1}, z_{1}, t_{1}$ such that

$$
0<Q\left(x_{1}, y_{1}, z_{1}, t_{1}\right)=\varepsilon<\varepsilon_{0},\left(x_{1}, y_{1}, z_{1}, t_{1}\right)=1 .
$$

By replacing $Q$ by an equivalent form we can suppose $Q(1,0,0,0)=\varepsilon$. Then $Q(x, y, z, t)$ can be written as

$$
Q(x, y, z, t)=\varepsilon(x+h y+g z+u t)^{2}-\varphi(y, z, t) ;
$$

where $\varphi(y, z, t)$ is an indefinite ternary quadratic form with determinant $-D / \varepsilon<0$. By Lemma 3 , we can find $(y, z, t) \equiv\left(y_{0}, z_{0}, t_{0}\right)(\bmod 1)$ such that

$$
0<\varphi(y, z, t)=\beta^{2} \leqq\left(\frac{4 D}{\varepsilon}\right)^{\frac{1}{3}} .
$$

Let $\alpha=h y+g z+u t$ and choose $x \equiv x_{0}(\bmod 1)$ with

so that

$$
\frac{\beta}{\sqrt{ } \varepsilon}<x+\alpha \leqq \frac{\beta}{\sqrt{ } \varepsilon}+1 \text {, }
$$




$$
\begin{aligned}
0<Q(x, y, z, t)=\varepsilon(x+\alpha)^{2}-\beta^{2} & \leqq \varepsilon+2 \beta \sqrt{ } \varepsilon \\
& \leqq \varepsilon+2\left(\frac{4 D}{\varepsilon}\right)^{\frac{1}{6}} \varepsilon^{\frac{1}{2}} \\
& <\varepsilon_{0}+2(4 D)^{\frac{1}{6}} \varepsilon_{0}^{\frac{1}{3}} .
\end{aligned}
$$

Since $\varepsilon_{0}$ can be chosen arbitrarily small, the right hand side of (3.2) can be made as small as we please and the lemma follows.

\subsection{Proof Continued}

LEMMA 9. If $Q(x, y, z, t) \sim m Q_{1}=m(x y+z t)$, then the theorem is true. Equality is needed for $Q_{1}$ if and only if $\left(x_{0}, y_{0}, z_{0}, t_{0}\right) \equiv(0,0,0,0)(\bmod 1)$.

Proof. Without loss of generality we can suppose that

$$
Q=Q_{1}=x y+z t \text {. }
$$

Take any $(z, t) \equiv\left(z_{0}, t_{0}\right)(\bmod 1)$. Choose $y \equiv y_{0}(\bmod 1)$ with $0<y \leqq 1$ and then take $x \equiv x_{0}(\bmod 1)$ to satisfy

$$
0<Q(x, y, z, t)=x y+z t \leqq y \leqq 1=(16 D)^{\frac{1}{4}} .
$$

Equality can occur only if $y_{0} \equiv 0(\bmod 1)$. By symmetry for equality we must have

$$
x_{0} \equiv y_{0} \equiv z_{0} \equiv t_{0} \equiv 0(\bmod 1) \text {. }
$$

Clearly equality is necessary when $\left(x_{0}, y_{0}, z_{0}, t_{0}\right) \equiv(0,0,0,0)(\bmod 1)$. This completes the proof of the Lemma.

From now on we can suppose $m>0$ and

$$
Q \nsim m Q_{1}=m(x y+z t) .
$$

Then given $0<\varepsilon_{0}<\frac{1}{16}$, we can find integers $x_{1}, y_{1}, z_{1}, t_{1}$ to satisfy

$$
Q\left(x_{1}, y_{1}, z_{1}, t_{1}\right)=\frac{m}{1-\varepsilon} \leqq\left(\frac{81}{16} D\right)^{\frac{1}{t}} ; \quad 0 \leqq \varepsilon<\varepsilon_{0} ;
$$

by Lemma 1 .

By definition of $m$ we must have $\left(x_{1}, y_{1}, z_{1}, t_{1}\right)=1$; since $1-\varepsilon>\frac{1}{4}$. By a suitable unimodular transformation we can suppose that $Q(1,0,0,0)=$ $m / 1-\varepsilon . \quad Q(x, y, z, t)$ can then be written as

$$
Q(x, y, z, t)=\frac{m}{1-\varepsilon}\left\{(x+h y+g z+u t)^{2}-\varphi(y, z, t)\right\}
$$

where $\varphi(y, z, t)$ is an indefinite ternary quadratic form of determinant

$$
D_{1}=-\frac{D}{\left(\frac{m}{1-\varepsilon}\right)^{4}} \leqq-\frac{16}{81}
$$


Also, for integers $x, y, z, t$ we have either $Q(x, y, z, t) \leqq 0$ or $Q(x, y, z, t) \geqq m$; i.e. either

$$
\begin{aligned}
& (x+h y+g z+u t)^{2}-\varphi(y, z, t) \leqq 0 \quad \text { or } \\
& (x+h y+g z+u t)^{2}-\varphi(y, z, t) \geqq 1-\varepsilon .
\end{aligned}
$$

Because of homogeneity it suffices to prove

THEOREM A. Let

$$
Q(x, y, z, t)=(x+h y+g z+u t)^{2}-\varphi(y, z, t)
$$

where $\varphi(y, z, t)$ is an indefinite ternary quadratic form of determinant

$$
D_{1}=-D \leqq-\frac{16}{81} \text {. }
$$

Let $0<\varepsilon_{0}<\frac{1}{16}$ be given arbitrarily small. Suppose that for integers $x, y, z, t$ we have either

$$
Q(x, y, z, t) \leqq 0 \quad \text { or } \quad Q(x, y, z, t) \geqq 1-\varepsilon
$$

where $0 \leqq \varepsilon<\varepsilon_{0}<\frac{1}{1 \cdot \text {. }}$. Let

$$
d=(16 D)^{\frac{1}{4}}
$$

so that from (3.5) we have $d \geqq \frac{4}{3}$. Then given any real numbers $x_{0}, y_{0}, z_{0}, t_{0}$ we can find $(x, y, z, t) \equiv\left(x_{0}, y_{0}, z_{0}, t_{0}\right)(\bmod 1)$ such that

$$
0<Q(x, y, z, t) \leqq d .
$$

Equality holds in (3.8) if and only if $Q=Q_{2}$ or $Q_{3}$.

\subsection{Proof of THEOREM A}

LEMMA 10 . Let $\alpha, \beta, d$ be real numbers with $d \geqq 1$. Then for any real $x_{0}$ there exists $x \equiv x_{0}(\bmod 1)$ satisfying

$$
0<(x+\alpha)^{2}-\beta^{2} \leqq d,
$$

provided

$$
\beta^{2}<\left(\frac{[d]}{2}\right)^{2}
$$

If $d$ is not an integer (3.9) is true with strict inequality. If $d$ is an integer a sufficient condition for (3.9) to be true with strict inequality is that

$$
\beta^{2}<\left(\frac{d-1}{2}\right)^{2} .
$$

This is Lemma 6 of my paper [4]. 
LEMMA 11. Suppose we can find $(y, z, t) \equiv\left(y_{0}, z_{0}, t_{0}\right)(\bmod 1)$ satisfying

$$
-\left(d-\frac{1}{4}\right)<\varphi(y, z, t)\left\{\begin{array}{l}
\leqq\left(\frac{d-1}{2}\right)^{2}, \text { if } d \text { is an integer } \\
<\left(\frac{[d]}{2}\right)^{2}, \text { if } d \text { is not an integer. }
\end{array}\right.
$$

Then for any $x_{0}$ there exists $x \equiv x_{0}(\bmod 1)$ such that

$$
0<Q(x, y, z, t) \leqq d .
$$

Further strict inequality in (3.12) implies strict inequality in (3.13).

Proof. If $-\left(d-\frac{1}{4}\right)<\varphi(y, z, t)<0$, choose $x \equiv x_{0}(\bmod 1)$ with $|x+h y+g z+u t| \leqq \frac{1}{2}$, so that

$$
0<Q(x, y, z, t)=(x+h y+g z+u t)^{2}-\varphi(y, z, t)<\frac{1}{4}+d-\frac{1}{4}=d .
$$

If $\varphi(y, z, t) \geqq 0$, the result follows from Lemma 10 with $\alpha=h y+g z+u t$ and $\beta^{2}=\varphi(y, z, t)$.

LEMMA 12. If $d>6$, then the theorem is true with strict inequality.

Proof. By Lemma 3, we can find $(y, z, t) \equiv\left(y_{0}, z_{0}, t_{0}\right)(\bmod 1)$ such that

$$
0<\varphi(y, z, t)<(4 D)^{\frac{1}{3}}=\left(\frac{1}{4} d^{4}\right)^{\frac{1}{3}} .
$$

Therefore (3.12) is satisfied with strict inequality if

$$
\left(\frac{1}{4} d^{4}\right)^{\frac{1}{3}}<\left(\frac{d-1}{2}\right)^{2}
$$

or

$$
f(d)=d^{3}-7 d^{2}+3 d-1>0
$$

which is clearly true for $d \geqq 7$. If $6<d<7$, then (3.12) is satisfied if we have $\left(\frac{1}{4} d^{4}\right)^{\frac{1}{3}}<9$ or $d^{2}<54$, which is true for $d<7$. Thus $(3.12)$ is satisfied and the result follows from Lemma 11 .

LEMMA 13. If $3<d \leqq 6$, then again the theorem is true with strict inequality.

Proof. By Lemma 4, we can find $(y, z, t) \equiv\left(y_{0}, z_{0}, t_{0}\right)(\bmod 1)$ such that

$$
|\varphi(y, z, t)| \leqq\left(\frac{27}{100}|D|\right)^{\frac{1}{3}}=\left(\frac{27}{1600} d^{4}\right)^{\frac{1}{3}} .
$$

Now $\left(\frac{27}{1600} d^{4}\right)^{\frac{1}{3}}<d-\frac{1}{4}$, if

$$
f(d)=\frac{(4 d-1)^{3}}{d^{4}}>\frac{27}{25}
$$


Since $f^{\prime}(d)<0$ for $d>1, f(d)$ is a decreasing function of $d$. Therefore for $3<d \leqq 6, f(d) \geqq f(6)=\frac{23^{3}}{6^{4}}>\frac{27}{25}$. Also

$$
\left(\frac{27}{1600} d^{d}\right)< \begin{cases}\left(\frac{d-1}{2}\right)^{2} & \text { if } 4 \leqq d \leqq 6 \\ \left(\frac{[d]}{2}\right)^{2} & \text { if } 3<d<4\end{cases}
$$

can be easily verified to be true. Thus $\varphi(y, z, t)$ satisfies (3.12) and the result follows from Lemma 11 .

LEMMA 14. If $\varphi(y, z, t) \sim \rho\left(y^{2}+z t\right), \rho>0, d \leqq 3$, then again (3.8) holds with strict inequality.

Proof. Without loss of generality we can suppose

so that

$$
\varphi(y, z, t)=\rho\left(y^{2}+z t\right), \quad \rho>0
$$

$$
Q(x, y, z, t)=(x+h y+g z+u t)^{2}-\rho\left(y^{2}+z t\right) .
$$

By replacing $x$ by $x+\alpha y+\beta z+\gamma t$ where $\alpha, \beta, \gamma$ are suitable integers we can suppose that

$$
|h| \leqq \frac{1}{2}, \quad|g| \leqq \frac{1}{2}, \quad|u| \leqq \frac{1}{2} .
$$

We first assert that $h=g=u=0$. If $u \neq 0$, then

$$
0<Q(0,0,0,1)=u^{2} \leqq \frac{1}{4}<1-\varepsilon,
$$

contrary to (3.6). Similarly $g=0$. If $h \neq 0$, then

$$
0<Q(0,1,1,-1)=h^{2} \leqq \frac{1}{4}<1-\varepsilon,
$$

contrary to (3.6). Therefore,

$$
Q(x, y, z, t)=x^{2}-\rho\left(y^{2}+z t\right) .
$$

Choose any $(x, y) \equiv\left(x_{0}, y_{0}\right)(\bmod 1)$. Choose $z \equiv z_{0}(\bmod 1)$ with $0<z \leqq 1$. Now choose $t \equiv t_{0}(\bmod 1)$ to satisfy

$$
0<x^{2}-\rho y^{2}-\rho z t \leqq \rho z \leqq \rho=(4 D)^{\frac{1}{3}}=\left(\frac{1}{4} d^{4}\right)^{\frac{1}{3}}<d,
$$

since $d \leqq 3<4$. This proves the Lemma.

\subsection{Proof of theorem A continued}

From now on we can suppose that

$$
\frac{4}{3} \leqq d \leqq 3 ; \quad \varphi(y, z, t) \nsim \rho\left(y^{2}+z t\right), \quad \rho>0 .
$$

By Lemma 2, we can find integers $y_{2}, z_{2}, t_{2}$ such that $\left(y_{2}, z_{2}, t_{2}\right)=1$ and 


$$
0<a=\varphi\left(y_{2}, z_{2}, t_{2}\right) \leqq\left(\frac{9}{4} D\right)^{\frac{1}{3}}=\left(\frac{9}{64} d^{4}\right)^{\frac{1}{3}}
$$

By a unimodular transformation we can suppose that

$$
\varphi(y, z, t)=a\left\{(y+f z+v t)^{2}+\psi(z, t)\right\},
$$

where $\psi(z, t)$ is an indefinite binary quadratic form with discriminant

$$
\Delta^{2}=\frac{4 D}{a^{3}}=\frac{d^{4}}{4 a^{3}} \text {. }
$$

Without loss of generality we can also suppose that

$$
|h| \leqq \frac{1}{2}, \quad|g| \leqq \frac{1}{2}, \quad|u| \leqq \frac{1}{2}, \quad|f| \leqq \frac{1}{2}, \quad|v| \leqq \frac{1}{2} .
$$

In view of Lemma 11, if we can show that there exist $(y, z, t) \equiv\left(y_{0}, z_{0}, t_{0}\right)$ (mod 1) satisfying

$$
\text { (A) }-\left(d-\frac{1}{4}\right)<\varphi(y, z, t)=a\left\{(y+f z+v t)^{2}+\psi(z, t)\right\}\left\{\begin{array}{l}
<1 \text { if } 2<d \leqq 3 \\
\leqq \frac{1}{4} \text { if } \frac{4}{3} \leqq d \leqq 2
\end{array}\right.
$$

then the proof of Theorem A will be complete except for the equality part.

LEMMA 15. If $2<d \leqq 3$, then again the theorem is true with strict inequality.

Proof. Since $d \leqq 3$, we have from (3.15)

Let

$$
0<a \leqq\left(\frac{9}{64} d^{4}\right)^{\frac{1}{3}} \leqq \frac{9}{4} .
$$

$$
\lambda=\frac{4-a}{a \Delta},
$$

so that $\lambda>0$. By Lemma 5 , we can find $(z, t) \equiv\left(z_{0}, t_{0}\right)(\bmod 1)$ such that

$$
-\frac{d^{4}}{16 a^{2}(4-a)}=-\frac{a \Delta^{2}}{4(4-a)}=-\frac{\Delta}{4 \lambda} \leqq \psi(z, t)<\frac{\lambda \Delta}{4}=\frac{1}{a}-\frac{1}{4} .
$$

If

$$
-\frac{4 d-1}{4 a}<\psi(z, t)<\frac{1}{a}-\frac{1}{4}
$$

choose $y \equiv y_{0}(\bmod 1)$ with $|y+f z+v t| \leqq \frac{1}{2}$, so that

$$
-\left(d-\frac{1}{4}\right)<\varphi(y, z, t)=a\left\{(y+f z+v t)^{2}+\psi(z, t)\right\}<a\left(\frac{1}{4}+\frac{1}{a}-\frac{1}{4}\right)=1 .
$$

Thus (A) is satisfied and the result follows in this case. Let now

$$
\frac{4 d-1}{4 a} \leqq \beta=-\psi(z, t) \leqq \frac{d^{4}}{16 a^{2}(4-a)} .
$$


(A) will be satisfied if we can find $y \equiv y_{0}(\bmod 1)$ to satisfy

$$
\begin{gathered}
0<(y+f z+v t)^{2}-\left(\beta-\frac{4 d-1}{4 a}\right)<\frac{1}{a}+\frac{4 d-1}{4 a}=\frac{4 d+3}{4 a}, \\
\frac{4 d+3}{4 a}>1 ; \text { since } a \leqq \frac{9}{4}, \quad d>2 .
\end{gathered}
$$

In view of Lemma 10, this is possible if we have

$$
0 \leqq \beta-\frac{4 d-1}{4 a}<\left(\frac{\frac{4 d+3}{4 a}-1}{2}\right)^{2}
$$

This by (3.19) is possible if

$$
\frac{d^{4}}{16 a^{2}(4-a)}-\frac{4 d-1}{4 a}<\left(\frac{4 d+3-4 a}{8 a}\right)^{2} .
$$

A slight calculation shows that this is so if

$$
\begin{aligned}
f(a, d) & =a(13-4 d-4 a)^{2}+4\left\{d^{4}-(4 d+3)^{2}\right\}<0 . \\
\frac{\partial f}{\partial a} & =(13-4 d-4 a)(13-4 d-12 a) .
\end{aligned}
$$

Therefore, since $d \leqq \mathbf{3}$,

$$
\max f(a, d) \leqq \max \left\{f\left(\frac{13-4 d}{12}, d\right), f\left(\left(\frac{9}{64} d^{4}\right)^{\frac{1}{3}}, d\right)\right\},
$$

for

$$
a \leqq\left(\frac{9}{64} d^{4}\right)^{\frac{1}{3}} \leqq \max \left\{f\left(\frac{13-4 d}{12}, d\right), f\left(\frac{9}{4}, d\right)\right\} .
$$

For $2<d \leqq 3$,

and

$$
\begin{aligned}
f\left(\frac{13-4 d}{12}, d\right) & =\frac{(13-4 d)^{3}}{27}-4\left(d^{2}+4 d+3\right)\left(4 d+3-d^{2}\right) \\
& \leqq \frac{(13-8)^{3}}{27}-4(4+8+3)(4 \cdot 3+3-9) \\
& <0,
\end{aligned}
$$

$$
\begin{aligned}
f\left(\frac{9}{4}, d\right) & =4\left\{9(1-d)^{2}+d^{4}-(4 d+3)^{2}\right\} \\
& =4 d\left(d^{3}-7 d-42\right) \\
& <0 .
\end{aligned}
$$

Hence (3.20) is satisfied, so that (A) holds and the result follows. 
LEMMA 16. If $\frac{4}{3} \leqq d \leqq 2$, then again the theorem is true.

Proof. We shall distinguish the following three subcases:

(i) $1<a \leqq\left(\frac{9}{64} d^{4}\right)^{\frac{1}{3}}$

(ii) $0<a<1$

(iii) $a=1$.

Proof of (i). Let $\nu>1$ be a solution of

$$
f(v)=v^{4}-6 v^{2}+8 v-3-\frac{4 d^{4}}{a(a-1)^{2}}=0 .
$$

Such a $v$ exists, since $f(1)<0, f(\infty)>0$. Then

$$
\frac{\Delta}{\left\{(v-1)^{3}(v+3)\right\}^{\frac{1}{2}}}=\frac{a-1}{4 a} .
$$

By Lemma 7 , we can find $(z, t) \equiv\left(z_{0}, t_{0}\right)(\bmod 1)$ to satisfy

$$
-v^{2} \frac{(a-1)}{4 a} \leqq \psi(z, t)=-\beta<-\frac{a-1}{4 a} .
$$

If

$$
\frac{a-1}{4 a}<\beta<\frac{4 d-1}{4 a},
$$

choose $y \equiv y_{0}(\bmod 1)$ with $|y+f z+v t| \leqq \frac{1}{2}$, so that

$-\left(\frac{4 d-1}{4 a}\right)<-\beta \leqq(y+f z+v t)^{2}+\psi(z, t)=(y+f z+v t)^{2}-\beta<\frac{1}{4}-\frac{a-1}{4 a}=\frac{1}{4 a}$.

Thus (A) is satisfied and the result follows. Let now

$$
\frac{4 d-1}{4 a} \leqq \beta \leqq \frac{\nu^{2}(a-1)}{4 a} .
$$

In order that (A) be satisfied, we want to find $y \equiv y_{0}(\bmod 1)$ such that

$$
0<(y+f z+v t)^{2}-\left(\beta-\frac{4 d-1}{4 a}\right)<\frac{d}{a} .
$$

Since $1<a \leqq\left(\frac{9}{64} d^{4}\right)^{\frac{1}{3}}$ and $d \leqq 2$, we have

$$
\frac{1}{8}<\frac{a^{3}}{d^{3}} \leqq \frac{9 d}{64} \leqq \frac{9}{32}<1 .
$$

Therefore $1<d / a<2$, so that $[d / a]=1, d / a$ not an integer. By Lemma 10 , (3.22) will be satisfied if we have 


$$
\beta-\frac{4 d-1}{4 a}<\frac{1}{4}
$$

This by (3.21) will be so if

$$
\frac{v^{2}(a-1)}{4 a}<\frac{4 d+a-1}{4 a}
$$

or

$$
v^{2}<\frac{4 d+a-1}{a-1}=v_{0}^{2}, \quad \text { say }
$$

Since $f^{\prime}(v)=4(v-1)^{2}(v+2)>0, f(v)$ is an increasing function of $v$, and $f(1)<0$, it suffices to show that $f\left(v_{0}\right)>0$; or

$$
(4 d+a-1)^{2}-6(a-1)(4 d+a-1)+\left(8 v_{0}-3\right)(a-1)^{2}-\frac{4 d^{4}}{a}>0
$$

or

$$
8 a(a-1)^{2}\left(v_{0}-1\right)>4 d\left\{d^{3}-4 a d+4 a(a-1)\right\} .
$$

Since $a>1, v_{0}>1,(3.23)$ is clearly satisfied if we have

$$
\begin{aligned}
g(a, d) & =d^{3}-4 a d+4 a(a-1) \leqq 0 \\
\frac{\partial g}{\partial a} & =4(2 a-d-1) \\
& \leqq 4\left\{2\left(\frac{9}{64} d^{4}\right)^{\frac{1}{3}}-d-1\right\} \\
& =4\left\{\frac{3}{2} d\left(\frac{d}{3}\right)^{\frac{1}{3}}-d-1\right\} \\
& \left.<4\left\{\frac{3}{2} d-d-1\right\} \quad \text { (since } d \leqq 2\right) \\
& \leqq 0 .
\end{aligned}
$$

Therefore for $1<a \leqq\left(\frac{9}{64} d^{4}\right)^{\frac{1}{3}}$ and $\frac{4}{3} \leqq d \leqq 2$, we have

$$
g(a, d)<g(1, d)=d^{3}-4 d=d\left(d^{2}-4\right) \leqq 0 .
$$

Thus (3.24) is satisfied with strict inequality and the result follows. This proves the result in subcase (i).

Proof of (ii). Let

$$
\mu=-5+\left\{16+\frac{4 d^{4}}{a(1-a)^{2}}\right\}^{\frac{1}{2}}
$$

be a root of

$$
\frac{\Delta}{\{(1+\mu)(\mu+9)\}^{\frac{1}{2}}}=\frac{1-a}{4 a} .
$$

We have $\mu \geqq 3$, if $a(1-a)^{2} \leqq d^{4} / 12$, which is so, since 


$$
a(1-a)^{2} \leqq \frac{1}{3}\left(1-\frac{1}{3}\right)^{2}=\frac{4}{27}<\frac{d^{4}}{12},
$$

since $d \geqq \frac{4}{3}$. Thus by Lemma 6 , we can find $(z, t) \equiv\left(z_{0}, t_{0}\right)(\bmod 1)$ such that

$$
-\frac{\mu \Delta}{\{(1+\mu)(9+\mu)\}^{\frac{1}{2}}} \leqq \psi(z, t)<\frac{\Delta}{\{(1+\mu)(9+\mu)\}^{\frac{1}{2}}},
$$

or

$$
-\frac{\mu(1-a)}{4 a} \leqq \psi(z, t)<\frac{1}{4 a}-\frac{1}{4}
$$

If

$$
-\frac{4 d-1}{4 a}<\psi(z, t)<\frac{1}{4 a}-\frac{1}{4},
$$

choose $y \equiv y_{0}(\bmod 1)$, such that $|y+f z+v t| \leqq \frac{1}{2}$, so that

$$
-\left(d-\frac{1}{4}\right)<\varphi(y, z, t)=a\left\{(y+f z+v t)^{2}+\varphi(z, t)\right\}<\frac{1}{4} .
$$

Thus (A) is satisfied and the result follows. Let now

$$
\frac{4 d-1}{4 a} \leqq \beta=-\psi(z, t) \leqq \frac{\mu(1-a)}{4 a} .
$$

In order that $(A)$ be satisfied we want to choose $y \equiv y_{0}(\bmod 1)$ such that

$$
0<(y+f z+v t)^{2}-\left(\beta-\frac{4 d-1}{4 a}\right)<\frac{d}{a} .
$$

By Lemma 10, (3.26) will be satisfied if we have

$$
\beta-\frac{4 d-1}{4 a}<\left(\frac{d-a}{2 a}\right)^{2}
$$

This will be satisfied if we have

$$
\mu \frac{(1-a)}{4 a}-\frac{4 d-1}{4 a}<\left(\frac{d-a}{2 a}\right)^{2}
$$

Substituting for $\mu$ from (3.25), a slight simplification shows that the above is true if

$$
f(a, d)=16 a^{3}-4 a^{2}(4-d)-4 a d(2-d)(1+d)-d^{3}<0,
$$

for $0<a<1$.

By the rule of signs, for $\frac{4}{3} \leqq d \leqq 2, f(a, d)$ has at most one positive root. Since $f(\infty, d)>0$ and 


$$
\begin{aligned}
f(1, d) & =16-4(4-d)-4 d(2-d)(1+d)-d^{3} \\
& =3 d^{3}-4 d^{2}-4 d \\
& =d(3 d+2)(d-2) \\
& \leqq 0 \quad \text { for } \quad \frac{4}{3} \leqq d \leqq 2 .
\end{aligned}
$$

Thus for $0<a<1, \frac{4}{3} \leqq d \leqq 2$, we have

$$
f(a, d)<0 .
$$

The result then follows from Lemma 11.

Proof of (iii). $a=1$.

By Lemma 6 , with $\mu=\infty$, we can find $(z, t) \equiv\left(z_{0}, t_{0}\right)(\bmod 1)$ such that

$$
-\frac{d^{2}}{2}=-\Delta \leqq-\beta=\psi(z, t)<0
$$

by using (3.17).

We want to find $y \equiv y_{0}(\bmod 1)$ to satisfy $(\mathrm{A})$, i.e.

$$
-\frac{4 d-1}{4}<(y+f z+v t)^{2}-\beta \leqq \frac{1}{4} .
$$

If $0<\beta<\frac{1}{4}(4 d-1)$, then the result follows by choosing $y \equiv y_{0}(\bmod 1)$ with $\mid y+f z+v t) \leqq \frac{1}{2}$. Let now

$$
\frac{4 d-1}{4} \leqq \beta \leqq \frac{d^{2}}{2}
$$

(A) is equivalent to

$$
0<(y+f z+v t)^{2}-\left(\beta-\frac{4 d-1}{4}\right) \leqq d .
$$

By Lemma 10, (3.29) will be satisfied if we have

$$
\beta-\frac{4 d-1}{4} \leqq\left(\frac{d-1}{2}\right)^{2}
$$

From (3.28), the above will be true if

$$
\frac{d^{2}}{2}-\frac{4 d-1}{4} \leqq \frac{d^{2}-2 d+1}{4}
$$

or

$$
d \leqq 2 \text {, }
$$

which is so and hence the result follows from Lemma 11. This completes the proof of Lemma 16. 


\section{Case of equality}

LEMMA 17. Equality occurs if and only if $Q \sim Q_{2}$ or $Q_{3}$.

Proof. From Lemma 16, it follows that equality can occur only if

$$
a=1, \quad d=2, \quad \Delta^{2}=4 .
$$

Also we must have equality in Lemma 6 when $\mu=\infty$, so that either

$$
\begin{aligned}
& \psi(z, t) \sim c_{1}\left(z^{2}-t^{2}\right) ; \quad\left(z_{0}, t_{0}\right) \equiv\left(\frac{1}{2}, \frac{1}{2}\right)(\bmod 1) ; \quad \text { or } \\
& \psi(z, t) \sim c_{2} z t ; \quad\left(z_{0}, t_{0}\right) \equiv(0,0)(\bmod 1),
\end{aligned}
$$

where $c_{1}, c_{2}>0$. Since $\Delta^{2}=4$, we have $c_{1}=1, c_{2}=2$. Without loss of generality we can suppose that either

$$
\begin{array}{ll}
\psi(z, t)=z^{2}-t^{2} ; & \left(z_{0}, t_{0}\right) \equiv\left(\frac{1}{2}, \frac{1}{2}\right)(\bmod 1) ; \quad \text { or } \\
\psi\left(z_{2}, t_{2}\right)=2 z t ; & \left(z_{0}, t_{0}\right) \equiv(0,0)(\bmod 1) .
\end{array}
$$

We now discuss the two cases separately.

Case (i). $\psi(z, t)=z^{2}-t^{2} ;\left(z_{0}, t_{0}\right) \equiv\left(\frac{1}{2}, \frac{1}{2}\right)(\bmod 1)$.

If equality is to occur in $(A)$, then the inequalities

$$
\begin{aligned}
-\frac{7}{4}=-\frac{4 d-1}{4 a}<F(y, z, t)= & \left(y+f z+v t+y_{0}+\frac{f}{2}+\frac{v}{2}\right)^{2} \\
& +\left(z+\frac{1}{2}\right)^{2}-\left(t+\frac{1}{2}\right)^{2}<\frac{1}{4 a}=\frac{1}{4}
\end{aligned}
$$

should have no solution in integers $y, z, t$.

$$
-\frac{7}{4}<F(y, 0,0) \leqq\left(y+y_{0}+\frac{f}{2}+\frac{v}{2}\right)^{2}<\frac{1}{4}
$$

is solvable for integer $y$ unless

$$
y_{0}+\frac{t}{2}+\frac{v}{2} \equiv \frac{1}{2}(\bmod 1)
$$

Similarly by considering $F(y,-1,0)$ and $F(y, 0,-1)$ we find that if equality is to occur we must have

$$
y_{0}-\frac{f}{2}+\frac{v}{2} \equiv \frac{1}{2}(\bmod 1)
$$

and

$$
y_{0}+\frac{f}{2}-\frac{v}{2} \equiv \frac{1}{2}(\bmod 1)
$$


From (4.1), (4.2), (4.3) and (3.18) we get

$$
f=v=0, \quad y_{0} \equiv \frac{1}{2}(\bmod 1) .
$$

Thus if equality is to occur we must have

$$
\varphi(y, z, t)=y^{2}+z^{2}-t^{2}, \quad\left(y_{0}, z_{0}, t_{0}\right) \equiv\left(\frac{1}{2}, \frac{1}{2}, \frac{1}{2}\right)(\bmod 1) .
$$

Again, if equality is to occur, the inequalities

$$
\begin{gathered}
0<G(x, y, z, t)=\left(x+h y+g z+u t+x_{0}+\frac{h}{2}+\frac{g}{2}+\frac{u}{2}\right)^{2}-\left(y+\frac{1}{2}\right)^{2} \\
-\left(z+\frac{1}{2}\right)^{2}+\left(t+\frac{1}{2}\right)^{2}<2,
\end{gathered}
$$

should have no solution in integers $x, y, z, t$.

$$
0<G(x, 0,0,0)=\left(x+\frac{h}{2}+\frac{g}{2}+\frac{u}{2}+x_{0}\right)^{2}-\frac{1}{4}<2
$$

is solvable for integer $x$ unless

$$
\frac{h}{2}+\frac{g}{2}+\frac{u}{2}+x_{0} \equiv \frac{1}{2}(\bmod 1) .
$$

Similarly by considering $G(x, 0,0,-1), G(x, 0,-1,0)$ and $G(x,-1,0,0)$ we find that if equality is to occur we must have

$$
\begin{aligned}
\frac{h}{2}+\frac{g}{2}-\frac{u}{2}+x_{0} & \equiv \frac{1}{2}(\bmod 1), \\
\frac{h}{2}-\frac{g}{2}+\frac{u}{2}+x_{0} & \equiv \frac{1}{2}(\bmod 1), \\
-\frac{h}{2}+\frac{g}{2}+\frac{u}{2}+x_{0} & \equiv \frac{1}{2}(\bmod 1) .
\end{aligned}
$$

From (4.4), (4.5), (4.6), (4.7) and (3.18) we get

$$
h=g=u=0, \quad x_{0} \equiv \frac{1}{2}(\bmod 1) .
$$

Thus in case (i), equality can occur only if

$$
Q=x^{2}-y^{2}-z^{2}+t^{2}=Q_{2}, \quad\left(x_{0}, y_{0}, z_{0}, t_{0}\right) \equiv\left(\frac{1}{2}, \frac{1}{2}, \frac{1}{2}, \frac{1}{2}\right)(\bmod 1) .
$$

We next show that equality is needed for this form. For this it suffices to show that for integers $x, y, z, t$ we have either

i.e.

$$
\left(x+\frac{1}{2}\right)^{2}-\left(y+\frac{1}{2}\right)^{2}-\left(z+\frac{1}{2}\right)^{2}+\left(t+\frac{1}{2}\right)^{2} \leqq 0 \text { or } \geqq 2,
$$

$$
X^{2}-Y^{2}-Z^{2}+T^{2} \leqq 0 \text { or } \geqq 8
$$


for odd integers $X, Y, Z, T$. This is clearly so, since

$$
X^{2}-Y^{2}-Z^{2}+T^{2} \equiv 1-1-1+1 \equiv 0(\bmod 8)
$$

for odd integers $X, Y, Z, T$.

This completes the proof of the lemma in this case.

Case (ii). $\psi(z, t)=2 z t ;\left(z_{0}, t_{0}\right) \equiv(0,0)(\bmod 1)$.

If equality is to occur in (A), then the inequalities

$$
-\frac{7}{4}<F(y, z, t)=\left(y+f z+v t+y_{0}\right)^{2}+2 z t<\frac{1}{4}
$$

should have no solutions in integers $y, z, t$.

By considering $F(y, 0,0), F(y, 1,0)$ and $F(y, 0,1)$ we see that if equality is to occur we must have

$$
\begin{aligned}
y_{0} & \equiv \frac{1}{2}(\bmod 1), \\
y_{0}+f & \equiv \frac{1}{2}(\bmod 1), \\
y_{0}+v & \equiv \frac{1}{2}(\bmod 1) .
\end{aligned}
$$

From (4.9), (4.10), (4.11) and (3.18) we get

$$
f=v=0, \quad y_{0} \equiv \frac{1}{2}(\bmod 1) .
$$

Thus if equality is to occur we must have

$$
\varphi(y, z, t)=y^{2}+2 z t, \quad\left(y_{0}, z_{0}, t_{0}\right) \equiv\left(\frac{1}{2}, 0,0\right)(\bmod 1) .
$$

Again, for equality, the inequalities

$$
0<G(x, y, z, t)=\left(x+h y+g z+u t+\frac{h}{2}+x_{0}\right)^{2}-\left(y+\frac{1}{2}\right)^{2}-2 z t<2
$$

should have no solution in integers $x, y, z, t$. By considering $G(x, 0,0,0)$, $G(x, 0,0,1), G(x, 0,1,0)$ and $G(x,-1,0,0)$ we see that if equality is to occur we must have

$$
\begin{aligned}
x_{0}+\frac{h}{2} & \equiv \frac{1}{2}(\bmod 1), \\
x_{0}-\frac{h}{2} & \equiv \frac{1}{2}(\bmod 1), \\
x_{0}+\frac{h}{2}+g & \equiv \frac{1}{2}(\bmod 1),
\end{aligned}
$$

and

$$
x_{0}+\frac{h}{2}+u \equiv \frac{1}{2}(\bmod 1) \text {. }
$$


From (4.12), (4.13), (4.14), (4.15), and (3.18) we have

$$
h=g=u=0, \quad x_{0} \equiv \frac{1}{2}(\bmod 1) \text {. }
$$

Thus equality can occur only if

$$
Q(x, y, z, t)=x^{2}-y^{2}-2 z t=Q_{3}, \quad\left(x_{0}, y_{0}, z_{0}, t_{0}\right) \equiv\left(\frac{1}{2}, \frac{1}{2}, 0,0\right)(\bmod 1) .
$$

We next show that equality is needed for this form. For this it suffices to show that for integers $x, y, z, t$ we have either

$$
\begin{aligned}
& \left(x+\frac{1}{2}\right)^{2}-\left(y+\frac{1}{2}\right)^{2}-2 z t \leqq 0 \quad \text { or } \geqq 2, \quad \text { i.e. } \\
& (2 x+1)^{2}-(2 y+1)^{2}-8 z t \leqq 0 \text { or } \geqq 8 .
\end{aligned}
$$

This is obviously true, since left hand side is $\equiv 0(\bmod 8)$ for integers $x, y, z, t$. This completes the proof of the Lemma and the theorem follows.

\section{References}

[1] E. S. Barnes, 'The positive values of inhomogeneous ternary quadratic forms', J. A ustralian Math. Soc. 2 (1961), $127-132$.

[2] H. Blaney, 'Some asymmetric inequalities', Proc. Camb. Phil. Soc. 46 (1950), 359-376.

[3] H. Davenport, 'Non-homogeneous ternary quadratic forms,' Acta Math. 80 (1948), $65-95$.

[4] V. C. Dumir, 'Positive values of inhomogeneous quaternary quadratic forms, I', Jour. Aust. Math. Soc. 8 (1968), 87-101.

[5] A. Oppenheim, 'One sided inequalities for quadratic forms (I), Ternary forms', Proc. London Math. Soc. (3) 3 (1953), 328-337.

[6] A. Oppenheim, 'One sided inequalities for quadratic forms (II). Quaternary forms', Proc. London Math. Soc. (3) 3 (1953), 417-429.

University of Illinois

Urbana, Illinois 